\title{
Spinors and Rodrigues Representations Associated with Orthogonal Polynomials
}

\author{
Zahra Bakhshi \\ Department of Physics, Faculty of Basic Sciences, Shahed University, Tehran, Iran \\ Correspondence should be addressed to Zahra Bakhshi; z.bakhshi@shahed.ac.ir
}

Received 26 December 2017; Revised 18 March 2018; Accepted 26 March 2018; Published 20 May 2018

Academic Editor: Shi-Hai Dong

Copyright ( 2018 Zahra Bakhshi. This is an open access article distributed under the Creative Commons Attribution License, which permits unrestricted use, distribution, and reproduction in any medium, provided the original work is properly cited. The publication of this article was funded by $\mathrm{SCOAP}^{3}$.

\begin{abstract}
An effective approach is presented to produce Schrödinger-like equation for the spinor components from Dirac equation. Considering electrostatic potential as a constant value yields a second-order differential equation that is comparable with the wellknown solvable models in the nonrelativistic quantum mechanics for the certain bound state energy spectrum and the well-known potentials. By this comparison, the gauge field potential and the relativistic energy can be written by the nonrelativistic models and the spinors will be related to the orthogonal polynomials. It has also shown that the upper spinors wave functions based on the orthogonal polynomials can be given in terms of the Rodrigues representations. Association with the Rodrigues representations of orthogonal polynomials has also been investigated in the lower spinor components, since they are related to the upper spinor components according to first-order differential equation that is attained from Dirac equation.
\end{abstract}

\section{Introduction}

In recent years, there has been a developing interest in search for exactly solvable systems in nonrelativistic and relativistic quantum mechanics. The expression exactly solvable means the eigenvalues and the eigenfunctions of the Hamiltonian operator of the physical system can be derived analytically in closed form. Solvable models are noteworthy because understanding of physics can only be brought with such solutions. Moreover, exact solutions are valuable tools for testing and improving numerical methods introduced to solve problems physically more interesting [1]. Since relativistic extensions of the exact solvable potentials are very useful to study the relativistic effects, various methods were employed to obtain the exact solution of the problem. Point canonical transformation [2-4], dynamical group [5, 6], factorization method [7], supersymmetric quantum mechanics, and shape invariance [8-10] are methods among many which were used in the search for exact solutions of wave function. Also, there are a lot of investigations that show how methods used to obtain analytical solutions of the Schrödinger equation can be extended to Dirac case [11-15].
Alhaidari [11-13] applies a unitary transformation to Dirac equation such that the resulting second-order differential equation becomes Schrödinger-like equation so that comparison with the well-known nonrelativistic problems is transparent. If the electrostatic potential is assumed as a constant value, the second-order differential equation can be constituted for upper component by eliminating lower component, without applying a general local unitary transformation that eliminates the first-order derivative such as what Alhaidari has considered.

In this method, by assuming electrostatic potential as a constant value, the second-order differential equation can be compared with the well-known solvable Schrödinger equation in the nonrelativistic quantum models. The wave functions in Schrödinger equation for the well-known potentials have been obtained on the orthogonal polynomials, such as Jacobi, generalized Laguerre, and Hermite polynomials and the energy eigenvalues spectrum can be accessible for each case. By comparing the second-order differential equation that has been obtained from Dirac equation with Schrödinger equation for the well-known potential such as ScarffII, Pöshel-Teller, Mörse, 3D-oscillator, and shift-oscillator 
potentials, the gauge field potential can be written based on the well-known superpotentials that are related to the mentioned potentials. Therefore, the second-order differential is transformed to the solvable models with the exact solutions; it means that the relativistic energy eigenvalues can be gotten based on the nonrelativistic models, and also the spinors will be related to the orthogonal polynomials according to the nonrelativistic models. Then, Rodrigues representations and the differential equation of them are calculated for orthogonal polynomials. Moreover, the second-order differential equation also can be considered as a product of two firstorder differential operators and the spinor wave function related to the differential equation that is expressed in terms of Rodrigues representations related to the orthogonal polynomials. Therefore, the solution of second-order differential equation can be considered with the determined relativistic energy and association with Rodrigues representations can be gotten for each orthogonal polynomial.

This paper is organized as followed: In Section 2, by using the point canonical transformations, the second-order differential equation is constituted with the gauge field potential and the energy spectrum that will be introduced based on the nonrelativistic models. Then, the association of Rodrigues representation with orthogonal polynomials is shown in the Sections 3, 4, and 5 for Jacobi, generalized Laguerre, and Hermite polynomials, respectively. In each section, all of the gauge field potentials are considered to have the ability to constitute the solvable models with the certain energy eigenvalues, for each orthogonal polynomial. Therefore, in the each section, Rodrigues representations of the orthogonal polynomials have been calculated for some gauge field potentials. In Section 6, the paper ends with a brief conclusion.

\section{The Three-Dimensional Dirac Equation for a Free Structure}

Particle of spin $1 / 2$ reads $\left(i \hbar \gamma^{\mu} \partial_{\mu}-m c\right) \Phi=0$, where $m$ is the rest mass of the particle, $c$ is the speed of light, and $\Phi$ is a four-component wave function. The four matrices $\left\{\gamma^{\mu}\right\}_{\mu=0}^{3}$ are given the following standard representation [16]:

$$
\begin{aligned}
& \gamma^{0}=\left(\begin{array}{cc}
I & 0 \\
0 & -I
\end{array}\right), \\
& \vec{\gamma}=\left(\begin{array}{cc}
0 & \vec{\sigma} \\
-\vec{\sigma} & 0
\end{array}\right),
\end{aligned}
$$

where $I$ is the $2 \times 2$ unit matrix and $\sigma$ are the usual $2 \times 2$ Pauli spin matrices. In atomic units $(m=e=\hbar=1)$, Dirac equation reads $\left(i \gamma^{\mu} \partial_{\mu}-\alpha^{-1}\right) \Phi=0$, where $\alpha=\hbar / m c=1 / c$ is the Compton wavelength of the particle. In the presence of the electromagnetic potential, $A_{\mu}=\left(A_{0}, \overrightarrow{c A}\right)$, gauge invariant coupling to the charged spinor is accomplished by the minimal substitution $\partial_{\mu} \rightarrow \partial_{\mu}+i \alpha A_{\mu}$, which transforms free Dirac equation into

$$
\left[i \gamma^{\mu}\left(\partial_{\mu}+i \alpha A_{\mu}\right)-\alpha^{-1}\right] \Phi=0
$$

For time independent potential, (2) gives the following matrix representation of Dirac Hamiltonian (in units of $m c^{2}=\alpha^{2}$ ) [14]:

$$
H=\left(\begin{array}{cc}
\alpha^{2} A_{0}+1 & -i \alpha \vec{\sigma} \cdot \vec{\nabla}+\alpha \vec{\sigma} \cdot \vec{A} \\
-i \alpha \vec{\sigma} \cdot \vec{\nabla}+\alpha \vec{\sigma} \cdot \vec{A} & \alpha^{2} A_{0}-1
\end{array}\right) .
$$

Taking into consideration gauge invariance, the form of electromagnetic potential for static charge distribution with spherical symmetry is

$$
\left(A_{0}, \vec{A}\right)=(v(r), \widehat{r} \omega(r)),
$$

where $\widehat{r}$ is radial unit vector; $v(r)$ and $\omega(r)$ are electrostatic potential and gauge field potential, respectively. By substituting the two off-diagonal terms $\alpha \vec{\sigma} \cdot \vec{A}$ by $\pm i \alpha \vec{\sigma} \cdot \vec{A}$ in (3), the Hamiltonian leads to the following two-component radial Dirac equation [17]:

$$
\begin{aligned}
& \left(\begin{array}{cc}
\alpha^{2} v(r)+1 & \alpha\left(\frac{k}{r}+\omega(r)-\frac{d}{d r}\right) \\
\alpha\left(\frac{k}{r}+\omega(r)+\frac{d}{d r}\right) & \alpha^{2} v(r)-1
\end{array}\right)\left(\begin{array}{l}
\varphi(r) \\
\theta(r)
\end{array}\right) \\
& =\varepsilon\left(\begin{array}{l}
\varphi(r) \\
\theta(r)
\end{array}\right)
\end{aligned}
$$

where $\varepsilon$ are the relativistic energy eigenvalues and $k$ is the spin-orbit coupling parameter defined as $k= \pm(j+1 / 2)$ for $l=$ $j \pm 1 / 2$. Equation (5) gives two coupled first-order differential equations for the radial spinor components. By eliminating lower spinor component and by assuming the electrostatic potential $v(r)$ to be a constant value $\eta$, the second-order differential equation can be gotten for upper spinor wave function as

$$
\begin{gathered}
-\frac{d^{2} \varphi}{d r^{2}}+\left[\left(\omega(r)+\frac{k}{r}\right)^{2}-\left(\frac{d \omega}{d r}-\frac{k}{r^{2}}\right)\right. \\
\left.-\left(\frac{\left(\alpha^{2} \eta-\varepsilon\right)^{2}-1}{\alpha^{2}}\right)\right] \varphi(r)=0 .
\end{gathered}
$$

Equation (5) also gives the lower spinor component in terms of the upper component as follows:

$$
\theta(r)=\left[\alpha \eta-\left(\frac{\varepsilon+1}{\alpha}\right)\right]^{-1}\left\{\left[\omega(r)+\frac{k}{r}\right] \varphi(r)+\frac{d \varphi}{d r}\right\} .
$$

By comparing (6) with the solvable Schrödinger equation in the nonrelativistic models, the relation can be considered between the well-known potential in the nonrelativistic quantum models and the gauge field potential in the relativistic system as $V_{m}(r)=(\omega(r)+k / r)^{2}-\left(d \omega / d r-k / r^{2}\right)$. Also, nonrelativistic energy eigenvalues can be related to the relativistic energy eigenvalues as $E=\left(\left(\alpha^{2} \eta-\varepsilon\right)^{2}-1\right) / \alpha^{2}$. So, the gauge field potential and the relativistic energy due to solvability of Dirac equation based on the nonrelativistic quantum mechanics are easily available. 


\section{Association of Rodrigues Representation with Jacobi Polynomials}

Let us consider the gauge field potentials where their wave functions are related to Jacobi polynomials such as PöschlTeller potential $\omega^{(1)}(r)=-A \operatorname{coth} r+B / \sinh r-k / r$ and Scarf-II potential $\omega^{(2)}(r)=-A \tanh r-B / \cosh r-k / r$, where $A$ and $B$ are real parameters. For each potential, respectively, (6) gives the following second-order differential equations for upper spinor component:

$$
\begin{aligned}
- & \frac{d^{2} \varphi_{n, m}^{(1)}(r)}{d r^{2}} \\
+ & {\left[A^{2}+\frac{\left(B^{2}-A^{2}+A\right)}{\sinh ^{2} r}+\frac{(B-2 A B) \cosh r}{\sinh ^{2} r}\right] } \\
\cdot & \varphi_{n, m}^{(1)}(r)=\left[\frac{\left(\alpha^{2} \eta-\varepsilon\right)^{2}-1}{\alpha^{2}}\right] \varphi_{n, m}^{(1)}(r), \\
- & d^{2} \varphi_{n, m}^{(2)}(r) \\
d r^{2} & {\left[A^{2}+\frac{\left(B^{2}-A^{2}+A\right)}{\cosh ^{2} r}+\frac{(2 A B-B) \sinh r}{\cosh ^{2} r}\right] } \\
& \cdot \varphi_{n, m}^{(2)}(r)=\left[\frac{\left(\alpha^{2} \eta-\varepsilon\right)^{2}-1}{\alpha^{2}}\right] \varphi_{n, m}^{(2)}(r),
\end{aligned}
$$

where $A=(\lambda+\gamma+2 m-1) / 2$ and $B=(\gamma-\lambda) / 2$ such that $\lambda, \gamma>-1$ in (8) and $A=m+\lambda-1 / 2$ and $B=\gamma / 2$ such that $\lambda>-1$ and $-\infty>\gamma>+\infty$ in (9). There will be the well-known nonrelativistic energy spectrum as $E_{n, m}^{(1)}=(\lambda+$ $\gamma+n+m)(m-n-1)$ and $E_{n, m}^{(2)}=(2 \lambda+n+m)(n-m+1)$ for Pöschl-Teller potential and Scarf-II potential, respectively. As mentioned before, they can be used to calculate relativistic energy spectrum of Dirac equation as the following forms:

$$
\begin{aligned}
\varepsilon_{n}^{(1)}= & \alpha^{2} \eta \\
& \mp\left[-\alpha^{2}(\lambda+\gamma+n+m)(n-m+1)+1\right]^{1 / 2}, \\
\varepsilon_{n}^{(2)}= & \alpha^{2} \eta \mp\left[-\alpha^{2}(2 \lambda+n+m)(n-m+1)+1\right]^{1 / 2} .
\end{aligned}
$$

The bound states wave functions of the nonrelativistic problem [6] are mapped into the following upper spinor components wave functions:

$$
\begin{aligned}
& \varphi_{n, m}^{(1)}(x) \propto(x-1)^{(2 \lambda+2 m-1) / 4} \times(x+1)^{(2 \gamma+2 m-1) / 4} \\
& \cdot P_{n}^{(\lambda+m-1, \gamma+m-1)}(x), \\
& \varphi_{n, m}^{(2)}(x) \propto\left(1+x^{2}\right)^{-1 / 2} \times \exp \left(\frac{\gamma}{2} \tanh ^{-1} x\right) \\
& \cdot P_{n}^{(i(\gamma / 2)+m+\lambda-1 / 4,-i(\gamma / 2)+m+\lambda-1 / 4)}(x),
\end{aligned}
$$

where $P_{n}^{(\mu, \nu)}(x)$ is Jacobi polynomial with $\mu, \nu>-1$, and $x=$ $\cosh r, \mu=\lambda+m-1$, and $\nu=\gamma+m-1$ in (11) and $x=\sinh r$, $\mu+i(\gamma / 2)+m+\lambda-1 / 4$, and $\nu=-i(\gamma / 2)+m+\lambda-1 / 4$ in (12). By substituting upper spinor components (11) and (12) into (7) and using recursion properties of Jacobi polynomials, lower spinor components are given as

$$
\begin{aligned}
& \theta_{n, m}^{(1)}(x) \propto\left(\frac{1}{\alpha}\right. \\
& \left.\quad \mp\left[\frac{1}{\alpha^{2}}-n^{2}-n(\lambda+\gamma+2 m-1)\right]^{1 / 2}\right)^{-1} \\
& \quad \times\left(\left[n x-\left(\frac{\lambda-\gamma}{2 n+2 m+\lambda+\gamma-2}\right)\right]\left(x^{2}-1\right)^{-1 / 2}\right. \\
& \cdot \varphi_{n, m}^{(1)}(x)-\left[\frac{2(n+m+\lambda-1)(n+m+\gamma-1)}{2 n+2 m+\lambda+\gamma-2}\right] \\
& \left.\quad \cdot\left(x^{2}-1\right)^{-1 / 2} \varphi_{n-1, m}^{(1)}(x)\right), \\
& \theta_{n, m}^{(2)}(x) \propto\left(\frac{1}{\alpha}\right) \\
& \left.\quad+\left[\frac{1}{\alpha_{n-1, m}^{2}}+(n-m+1)(m-n+2 \lambda-2)\right]^{1 / 2}\right)^{-1} \\
& \quad \times\left(\left[-\left(n+\lambda+\frac{3}{4}\right) x+\frac{\gamma}{2}\left(\frac{2 n-3 m-\lambda+3}{-n+2 m-2}\right)\right]\right. \\
& \quad\left(x^{2}+1\right)^{-1 / 2}+\frac{\gamma}{2}(1-x)^{-1}\left(x^{2}+1\right)^{1 / 2} \varphi_{n, m}^{(2)}(x) \\
& \quad\left(x^{2}+1\right)^{-1 / 2}
\end{aligned}
$$

Raising and lowering operators $B_{ \pm}(m)= \pm d / d r+W_{m}(x(r))$, where the superpotential $W_{m}(x(r))$ satisfies in the Riccati equation $V_{m}=W_{m}^{2} \pm W_{m}^{\prime}$, can be written as the following forms according to Pöschl-Teller and Scarf-II potentials, respectively:

$$
\begin{aligned}
& B_{+}^{(1)}=\frac{d}{d r}+\left[-A \operatorname{coth} r+\frac{B}{\sinh r}\right], \\
& B_{-}^{(1)}=-\frac{d}{d r}+\left[-A \operatorname{coth} r+\frac{B}{\sinh r}\right], \\
& B_{+}^{(2)}=\frac{d}{d r}+\left[-A \tanh r-\frac{B}{\cosh r}\right], \\
& B_{-}^{(2)}=-\frac{d}{d r}+\left[-A \tanh r+\frac{B}{\cosh r}\right] .
\end{aligned}
$$


It is obvious that the second-order differential equations can always be considered in a factorization form as a product of a pair of linear differential operators (14) and (15). Therefore,

$$
\begin{gathered}
B_{+}^{(1),(2)}(m) B_{-}^{(1),(2)}(m) \varphi_{n, m}^{(1),(2)}(r) \\
=E^{(1),(2)}(n, m) \varphi_{n, m}^{(1),(2)}(r), \\
B_{-}^{(1),(2)}(m) B_{+}^{(1),(2)}(m) \varphi_{n, m-1}^{(1),(2)}(r) \\
=E^{(1),(2)}(n, m) \varphi_{n, m-1}^{(1),(2)}(r) .
\end{gathered}
$$

In the above equations, for a given $n$, the operator $B_{+}(m)$ raises the index $m$ while the operator $B_{-}(m)$ lowers it. We can also obtain the highest state $\varphi_{n, n}$ by solving the firstorder differential equation $B_{+}(n+1) \varphi_{n, n}(r)=0$ because the nonrelativistic energy spectrum $E(n, m)$ vanishes for $m=$ $n+1[10]$, since by introducing a new function as $\varphi_{n, m}(x)=$ $A^{1 / 4}(x) W^{1 / 2}(x) \psi_{n, m}(x)$ and changing the variable $d x / d r=$ $\sqrt{A(x)}$ Schrödinger equation (6) has been obtained from the general form associated with second-order differential equation in terms of master function $A(x)$ and the wave function $W(x)$ as follows [18-20]:

$$
\begin{aligned}
& A(x) \ddot{\psi}_{n, m}(x)+\frac{(A(x) W(x))^{\prime}}{W(x)} \dot{\psi}_{n, m}(x) \\
& +\left[-\frac{1}{2}\left(n^{2}+n-m^{2}\right) \ddot{A}(x)\right. \\
& +(m-n)\left(\frac{A(x) \dot{W}(x)}{W(x)}\right)^{\prime}-\frac{m^{2}}{4} \frac{\dot{A}(x)^{2}}{A(x)} \\
& \left.-\frac{m}{2} \frac{\dot{A}(x) \dot{W}(x)}{W(x)}\right] \psi_{n, m}(x)=0,
\end{aligned}
$$

where $\psi_{n, m}(x)$ is Rodrigues representation of the orthogonal polynomials in (17). For a positive integer $n, \psi_{n, m}(x)$ as Rodrigues representation is given by

$$
\begin{aligned}
\psi_{n, m}(x)=(-1)^{m} A^{m / 2}(x)\left(\frac{d}{d x}\right)^{m} \psi_{n}(x), & \\
& m=0,1,2, \ldots, n,
\end{aligned}
$$

where

$$
\psi_{n}(x)=\frac{N}{W(x)}\left(\frac{d}{d x}\right)^{n}\left(A^{n}(x) W(x)\right),
$$

with $N$ which is normalization constant. So, for each case of the gauge field potentials, the Rodrigues representations of upper and lower spinors and differential equations associated with them are available. Furthermore, it can be shown that they are clear examples of connection between Jacobi polynomials and Rodrigues representations in Dirac equation. Thus, the wave functions $\psi_{n, m}(x)$ are related to the upper spinors as the following forms:

$$
\psi_{n, m}^{(1)}(x)=(x-1)^{-(\lambda / 2+1 / 4)}(x+1)^{-(\gamma / 2+1 / 4)} \varphi_{n, m}^{(1)}(x),
$$

for $A^{(1)}(x)=x^{2}-1$ and $W^{(1)}(x)=(x-1)^{\lambda}(x+1)^{\gamma}$ in PöschlTeller potential and

$$
\begin{aligned}
& \psi_{n, m}^{(2)}(x) \\
& =\left(x^{2}+1\right)^{-(\lambda / 2+1 / 4)} \exp \left(-\frac{\gamma}{2} \tan ^{-1}(x)\right) \varphi_{n, m}^{(2)}(x),
\end{aligned}
$$

when $A^{(2)}(x)=x^{2}+1$ and $W^{(2)}(x)=\left(x^{2}+1\right)^{\lambda} \exp \left(\gamma \tan ^{-1}(x)\right)$ in Scarf-II potential. Since the lower spinors can be connected to the upper spinors according to (13), therefore, Rodrigues representations of upper spinors also can be associated with lower spinors. If the wave function $\Theta_{n, m}(x)$ is introduced for lower spinor $\theta_{n, m}(x)$, it can be written based on Rodrigues representations $\psi_{n, m}(x)$. The wave function $\Theta_{n, m}(x)$ that is connected to the lower spinors $\theta_{n, m}(x)$ can be written as follows for Pöschl-Teller and Scarf-II potentials, proportionately:

$$
\begin{aligned}
& \Theta_{n, m}^{(1)}(x) \propto\left(\frac{1}{\alpha}\right. \\
& \left.\mp\left[\frac{1}{\alpha^{2}}-n^{2}-n(\lambda+\gamma+2 m-1)\right]^{1 / 2}\right)^{-1} \\
& \times\left(\left[n x-\left(\frac{\lambda-\gamma}{2 n+2 m+\lambda+\gamma-2}\right)\right]\left(x^{2}-1\right)^{-1 / 2}\right. \\
& \cdot \psi_{n, m}^{(1)}(x)-\left[\frac{2(n+m+\lambda-1)(n+m+\gamma-1)}{2 n+2 m+\lambda+\gamma-2}\right] \\
& \left.\cdot\left(x^{2}-1\right)^{-1 / 2} \psi_{n-1, m}^{(1)}(x)\right), \\
& \Theta_{n, m}^{(2)}(x) \propto\left(\frac{1}{\alpha}\right. \\
& \left.\left.\quad \psi_{n-1, m}^{(2)}(x)\right) \cdot\left[\frac{i(-n+2 m+\lambda-2)^{2}+\gamma^{2} / 8}{\alpha^{2}}+(n-m+1)(m-n+2 \lambda-2)\right]^{1 / 2}\right)^{-1} \\
& \times\left(\left[-\left(x^{2}+1\right)^{-1 / 2}\right.\right. \\
& \quad\left(x^{2}+1\right)^{-1 / 2}+\frac{\gamma}{2}(1-x)^{-1}\left(x^{2}+1\right)^{1 / 2} \psi_{n, m}^{(2)}(x) \\
& \left.\quad\left[\frac{3}{4}\right) x+\frac{\gamma}{2}\left(\frac{2 n-3 m-\lambda+3}{-n+2 m-2}\right)\right]
\end{aligned}
$$

The wave functions $\psi_{n, m}(x)$ can be also satisfied in the second-order differential equations for each potential, correlatively:

$$
\begin{aligned}
& \left(x^{2}-1\right) \ddot{\psi}_{n, m}^{(1)}(x)+\left[\frac{1}{2}(1-\gamma-\lambda) x^{3}+\frac{1}{2}(\gamma-\lambda) x^{2}\right. \\
& -1] \dot{\psi}_{n, m}^{(1)}(x)+\left[(1-m)(\lambda+\gamma+m) x^{2}\right.
\end{aligned}
$$




$$
\begin{aligned}
& +(m-2)(\gamma-\lambda) x+\frac{(\lambda+\gamma+n+m)(m-n-1)}{x^{2}-1} \\
& \left.+\frac{(\lambda+\gamma+2 m-1)^{2}}{2}-m+1\right] \psi_{n, m}^{(1)}(x)=0, \\
& \left(x^{2}+1\right) \ddot{\psi}_{n, m}^{(2)}(x)+[2(\lambda+1) x+\gamma] \dot{\psi}_{n, m}^{(2)}(x) \\
& +\left[\left(m^{2}-n^{2}-n\right)+2 \lambda(m-n)\right. \\
& \left.+\frac{\left(m^{2}+2 m \lambda\right) x^{2}-\gamma m x}{x^{2}+1}\right] \psi_{n, m}^{(2)}(x)=0 .
\end{aligned}
$$

Rodrigues representations of the associated polynomials $\psi_{n, m}(x)$ are given by

$$
\begin{aligned}
\psi_{n, m}^{(1)}(x) & =(-1)^{m}\left(x^{2}-1\right)^{m / 2}\left(\frac{d}{d x}\right)^{m} \psi_{n}^{(1)}(x), \\
m & =0,1,2, \ldots, n, \\
\psi_{n, m}^{(2)}(x) & =(-1)^{m}\left(x^{2}+1\right)^{m / 2}\left(\frac{d}{d x}\right)^{m} \psi_{n}^{(2)}(x), \\
m & =0,1,2, \ldots, n,
\end{aligned}
$$

where $\psi_{n}(x)$ satisfies in Jacobi differential equation whose Rodrigues representations, respectively, are

$$
\begin{aligned}
& \psi_{n}^{(1)}(x)=N(x-1)^{-\lambda}(x+1)^{-\gamma}\left(\frac{d}{d x}\right)^{n} \\
& \cdot\left((x-1)^{n+\lambda}(x+1)^{n+\gamma}\right), \\
& \psi_{n}^{(2)}(x)=N\left(x^{2}+1\right)^{-\lambda} \exp \left(-\gamma \tan ^{-1} x\right)\left(\frac{d}{d x}\right)^{n} \\
& \cdot\left(\left(x^{2}+1\right)^{n+\lambda} \exp \left(-\gamma \tan ^{-1} x\right)\right),
\end{aligned}
$$

where $N$ is a normalization constant. Since the wave functions $\Theta_{n, m}(x)$ connected to the lower spinor $\theta_{n, m}(x)$ that have been calculated based on upper spinors $\varphi_{n, m}(x)$ and Rodrigues representations of upper spinors can be generalized to the wave function $\Theta_{n, m}(x)$ as $\psi_{n, m}(x)$ and $\psi_{n-1, m}(x)$, therefore, the above Rodrigues representation can also be related to the lower spinor components.

\section{Association of Rodrigues Representation with Generalized Laguerre Polynomials}

When Mörse potential $\omega^{(1)}(r)=-(\gamma / 2) e^{-r}-m-\lambda / 2+$ $1 / 2-k / r$ and 3-dimensional oscillator potential $\omega^{(2)}(r)=$ $(\gamma / 4) r-(\lambda+m-1 / 2)(2 / r)-k / r$ are considered as the gauge field potentials, the upper spinor components are associated with generalized Laguerre polynomials. So, the second-order differential equations for the upper spinor components are written according to (6):

$$
\begin{gathered}
-\frac{d^{2} \varphi_{n, m}^{(1)}(r)}{d r^{2}}+\left[\frac{\gamma^{2}}{4} e^{-2 r}+\gamma\left(m+\frac{\lambda}{2}-1\right) e^{-r}\right] \\
\cdot \varphi_{n, m}^{(1)}(r)=\left[\frac{\left(\alpha^{2} \eta-\varepsilon\right)^{2}-1}{\alpha^{2}}\right] \varphi_{n, m}^{(1)}(r), \\
-\frac{d^{2} \varphi_{n, m}^{(2)}(r)}{d r^{2}}+\left[\frac{\gamma^{2}}{16} r^{2}\right. \\
\left.+\left(\lambda+m-\frac{1}{2}\right)\left(\lambda+m-\frac{3}{2}\right) \frac{1}{r^{2}} \frac{\gamma}{2}(\lambda+m)\right] \\
\cdot \varphi_{n, m}^{(2)}(r)=\left[\frac{\left(\alpha^{2} \eta-\varepsilon\right)^{(2)}-1}{\alpha^{2}}\right] \varphi_{n, m}^{(2)}(r) .
\end{gathered}
$$

According to the nonrelativistic energy spectrum $E_{n, m}^{(1)}=$ $-(n-m+1)(\lambda+n+m)$ and $E_{n, m}^{(2)}=\gamma(n-m+1)$, the relativistic energy spectrums are obtained as

$$
\begin{aligned}
& \varepsilon_{n}^{(1)}=\alpha^{2} \eta \mp\left[-\alpha^{2}(n-m+1)(\lambda+n+m)+1\right]^{1 / 2}, \\
& \varepsilon_{n}^{(2)}=\alpha^{2} \eta \mp\left[-\alpha^{2} \gamma(n-m+1)+1\right]^{1 / 2} .
\end{aligned}
$$

Second-order differential equations (28) are due to the solutions based on the generalized Laguerre polynomials as upper spinor wave functions

$$
\begin{aligned}
\varphi_{n, m}^{(1)}(x) \propto & \left(\frac{\gamma}{x}\right)^{-(n+\lambda / 2+1 / 2)} \\
& \times \exp \left(-\frac{\gamma}{2 x}\right) L_{n}^{(-2 n-\lambda-1)}\left(\frac{\gamma}{x}\right), \\
\varphi_{n, m}^{(2)}(x) \propto & (\gamma x)^{(\lambda+m-1 / 2) / 2} \\
& \times \exp \left(-\frac{\gamma x}{2}\right) L_{n}^{(\lambda+m-1 / 4)}(\gamma x),
\end{aligned}
$$

where $L_{n}^{\alpha}(x)$ is generalized Laguerre polynomial with $\alpha>-1$. In the upper spinor (30), $x=e^{r}$ and $\alpha=-2 n-\lambda-1$ and, in the other upper spinor (31), $x=r^{2} / 4$ and $\alpha=\lambda+m-1 / 4$. Lower spinor components can be attained by (7) for each potential

$$
\begin{aligned}
& \theta_{n, m}^{(1)}(x) \propto\left(\frac{1}{\alpha}\right. \\
& \left.\mp\left[\frac{1}{\alpha^{2}}+(n-m+1)(-m-n-\lambda)\right]^{1 / 2}\right)^{-1} \\
& \quad \times\left[\frac{(-2 m-\lambda+1)-(n-m+1)}{\gamma}\right] x \varphi_{n-1, m}^{(1)}(x),
\end{aligned}
$$




$$
\begin{aligned}
& \theta_{n, m}^{(2)}(x) \propto\left(\frac{1}{\alpha} \mp\left[\frac{1}{\alpha^{2}}+\gamma(n-m+1)\right]^{1 / 2}\right)^{-1} \\
& \quad \times x^{-1 / 2}\left[(n-m+1) \varphi_{n, m}^{(2)}(\gamma x)\right. \\
& \left.-\left(n+\lambda+\frac{3}{4}\right) \varphi_{n-1, m}^{(2)}(\gamma x)\right] .
\end{aligned}
$$

As mentioned in pervious section, the raising and lowering $B_{+}$and $B_{-}$operators based on the superpotentials are given as

$$
\begin{aligned}
& B_{+}^{(1)}=\frac{d}{d r}-\frac{\gamma}{2} e^{-r}-m-\frac{\lambda}{2}+\frac{1}{2}, \\
& B_{-}^{(1)}=-\frac{d}{d r}-\frac{\gamma}{2} e^{-r}-m-\frac{\lambda}{2}+\frac{1}{2}, \\
& B_{+}^{(2)}=\frac{d}{d r}+\frac{\gamma}{4} r-\left(\lambda+m-\frac{1}{2}\right) \frac{2}{r}, \\
& B_{-}^{(2)}=-\frac{d}{d r}+\frac{\gamma}{4} r-\left(\lambda+m-\frac{1}{2}\right) \frac{2}{r} .
\end{aligned}
$$

The pair of linear differential operators can factorize Schrödinger equation for each potential. Similar to the pervious section, in Mörse potential, if $A^{(1)}(x)=x^{2}$ and $W^{(1)}(x)=x^{\lambda} e^{-\gamma / x}$, the wave function $\psi_{n, m}^{(1)}(x)$ is written based on upper spinor as

$$
\psi_{n, m}^{(1)}(x)=\left(\frac{\exp (\gamma / 2 x)}{x}\right) \varphi_{n, m}^{(1)}(x),
$$

and, in 3-dimensional oscillator potential, when $A^{(2)}(x)=x$ and $W^{(2)}(x)=x^{\lambda} e^{-\gamma x}$, the wave function $\psi_{n, m}^{(2)}(x)$ is obtained as

$$
\psi_{n, m}^{(2)}(x)=x^{-(\lambda / 2+1 / 4)} \exp \left(\frac{\gamma x}{2}\right) \varphi_{n, m}^{(2)}(x) .
$$

Therefore, they are also related to the generalized Laguerre polynomials. It is clear that both of them are examples of associating generalized Laguerre polynomial with Rodrigues representation in Dirac equation. Also, Rodrigues representation of lower spinors $\theta_{n, m}(x)$ that are called $\Theta_{n, m}(x)$ will be in the following forms based on $\psi_{n, m}(x)$ and $\psi_{n-1, m}(x)$, for Mörse and 3-dimensional oscillator potentials:

$$
\begin{aligned}
& \Theta_{n, m}^{(1)}(x) \propto\left(\frac{1}{\alpha}\right. \\
& \left.\mp\left[\frac{1}{\alpha^{2}}+(n-m+1)(-m-n-\lambda)\right]^{1 / 2}\right)^{-1} \\
& \times x\left[-\frac{(m+n+\lambda)}{\gamma}\right] \psi_{n-1, m}^{(1)}(x), \\
& \Theta_{n, m}^{(2)}(x) \propto\left(\frac{1}{\alpha} \mp\left[\frac{1}{\alpha^{2}}+\gamma(n-m+1)\right]^{1 / 2}\right)^{-1} \\
& \quad \times x^{(\lambda+1) / 2\left[(n-m+1) \psi_{n, m}^{(2)}(x)\right.} \\
& \left.+\left(n+\lambda+\frac{3}{4}\right) \psi_{n-1, m}^{(2)}(x)\right] .
\end{aligned}
$$

The wave functions $\psi_{n, m}(x)$ can be also satisfied in secondorder differential equations for each potential, proportionately:

$$
\begin{aligned}
& x^{2} \ddot{\psi}_{n, m}^{(1)}(x)+(\lambda x+\gamma+2) \dot{\psi}_{n, m}^{(1)}(x)+\left(-\frac{m \gamma}{x}+m^{2}\right. \\
& \left.-m-2 n^{2}-n(\lambda+1)\right) \psi_{n, m}^{(1)}(x)=0, \\
& x \ddot{\psi}_{n, m}^{(2)}(x)+\left(2\left(\lambda+\frac{1}{2}\right)-\gamma x\right) \dot{\psi}_{n, m}^{(2)}(x) \\
& +\left(\gamma\left(n-m+\frac{1}{2}\right)-\frac{\gamma \lambda}{2}\right. \\
& \left.-\frac{(m-1)(2 \lambda+1)}{4} x^{-1 / 2}\right) \psi_{n, m}^{(2)}(x)=0 .
\end{aligned}
$$

Rodrigues representations of associated polynomials $\psi_{n, m}(x)$ are given as

$$
\begin{aligned}
\psi_{n, m}^{(1)}(x) & =(-1)^{m} x^{m}\left(\frac{d}{d x}\right)^{m} \psi_{n}^{(1)}(x), \\
m & =0,1,2, \ldots, n, \\
\psi_{n, m}^{(2)}(x)=(-1)^{m} x^{m / 2}\left(\frac{d}{d x}\right)^{m} \psi_{n}^{(2)}(x) & , \\
m & =0,1,2, \ldots, n,
\end{aligned}
$$

where $\psi_{n}(x)$ satisfies in Laguerre differential equation whose Rodrigues representations are, respectively,

$$
\begin{aligned}
& \psi_{n}^{(1)}(x) \\
& =N x^{-\lambda} \exp \left(\frac{\gamma}{x}\right)\left(\frac{d}{d x}\right)^{n}\left(x^{2 n+\lambda} \exp \left(-\frac{\gamma}{x}\right)\right), \\
& \psi_{n}^{(2)}(x)=N x^{-\lambda} \exp (\gamma x)\left(\frac{d}{d x}\right)^{n}\left(x^{\lambda+n} \exp (-\gamma x)\right) .
\end{aligned}
$$

The above Rodrigues representations can be also related to lower spinor components, because there are the wave function $\Theta_{n, m}(x)$ based on the wave function $\psi_{n, m}(x)$ and $\psi_{n-1, m}(x)$ according to the lower spinor components.

\section{Association of Rodrigues Representation with Hermite Polynomials}

The upper spinor component will be considered as Hermite polynomials, if the gauge field potential $\omega(r)=(\gamma / 2) r-\lambda-$ $k / r$ is written based on shift-oscillator potential. This upper spinor component satisfies in (6) as

$$
\begin{gathered}
-\frac{d^{2} \varphi_{n, m}(r)}{d r^{2}}+\left[\left(\frac{\gamma}{2} r-\lambda\right)^{2}-\frac{\gamma}{2}\right] \varphi_{n, m}(r) \\
=\left[\frac{\left(\alpha^{2} \eta-\varepsilon\right)^{2}-1}{\alpha^{2}}\right] \varphi_{n, m}(r) .
\end{gathered}
$$


For this potential, the nonrelativistic energy $E_{n, m}=\gamma(n-$ $m+1)$ can be used in the following relativistic energy spectrum:

$$
\varepsilon_{n}=\alpha^{2} \eta \mp\left[\alpha^{2} \gamma(n-m+1)+1\right]^{1 / 2} .
$$

The upper spinor wave function based on the Hermite polynomials can be obtained from (42):

$$
\varphi_{n, m}(x) \propto \exp \left(-\frac{\gamma}{4} x^{2}\right) \times H_{n}\left(\left(\frac{\gamma}{2}\right)^{1 / 2} x\right)
$$

where $H_{n}(x)$ is Hermite polynomial. In the wave function (44) $x=r-2 \lambda / \gamma$ and $-\infty<x<+\infty$. According to (7), the lower spinor wave function is calculated as

$$
\begin{aligned}
\theta_{n, m}(x) \propto & \left(\frac{1}{\alpha} \mp\left[\frac{1}{\alpha^{2}}+\gamma(n-m+1)\right]^{1 / 2}\right)^{-1} \\
& \times\left(\left[(2 \gamma)^{1 / 2}(n-m+1)\right] \varphi_{n-1, m}(x) .\right.
\end{aligned}
$$

To factorize Schrödinger equation, there are pairs of linear differential operator as

$$
\begin{aligned}
& B^{+}=\frac{d}{d r}+\frac{\gamma}{2} r-\lambda, \\
& B^{-}=-\frac{d}{d r}+\frac{\gamma}{2} r-\lambda .
\end{aligned}
$$

As mentioned before, the wave function $\psi_{n, m}(x)$ that is related to upper spinor is gotten by

$$
\psi_{n, m}(x)=\exp \left(\frac{\gamma}{4} x^{2}\right) \varphi_{n, m}(x),
$$

for $A(x)=1$ and $W(x)=\exp \left(-(\gamma / 2) x^{2}\right)$. In this potential, Hermite polynomial can be associated with Rodrigues representation in Dirac equation. Also, similar to pervious section, the wave function $\Theta_{n, m}(x)$ that is connected to lower spinor $\theta_{n . m}(x)$ can be written based on Rodrigues representation:

$$
\begin{aligned}
\Theta_{n, m}(x) \propto & \left(\frac{1}{\alpha} \mp\left[\frac{1}{\alpha^{2}}+\gamma(n-m+1)\right]^{1 / 2}\right)^{-1} \\
& \times\left[(2 \gamma)^{1 / 2}(n-m+1)\right] \psi_{n-1, m}(x) .
\end{aligned}
$$

The second-order differential equation for shift-oscillator potential will be

$$
\ddot{\psi}_{n, m}(x)+(-\gamma x) \dot{\psi}_{n, m}(x)-\gamma(m-n) \psi_{n, m}(x)=0,
$$

where Rodrigues representation of the associated polynomial $\psi_{n, m}(x)$ is considered by

$$
\psi_{n, m}(x)=(-1)^{m}\left(\frac{d}{d x}\right)^{m} \psi_{n}(x), \quad m=0,1,2, \ldots, n
$$

and Rodrigues representation of $\psi_{n}(x)$, will be in the following form:

$$
\psi_{n}(x)=N \exp \left(\frac{\gamma}{2} x^{2}\right)\left(\frac{d}{d x}\right)^{n}\left(\exp \left(-\frac{\gamma}{2} x^{2}\right)\right) .
$$

Since $\Theta_{n, m}(x)$ has been used for lower spinor $\Theta_{n, m}(x)$ and it has been associated with $\psi_{n-1, m}(x)$ according to (48), therefore, (49), (50), and (51) can be considered for the lower spinor component in shift-oscillator potential.

\section{Conclusion}

A procedure for connecting the methods used in the analysis of exactly solvable potentials in the nonrelativistic quantum mechanics with the solution of Dirac equation has presented. A gauge field potential and the bound states energy spectrum have been defined for the Dirac equation with a constant electrostatic potential that can be constituted by a Schrödingerlike equation. Since orthogonal polynomials are considered as the solution of Schrödinger-like equation that have been obtained from Dirac equation, Rodrigues representations of the orthogonal polynomials can be associated with upper and lower spinor components.

\section{Conflicts of Interest}

The author declares that she has no conflicts of interest.

\section{References}

[1] A. D. Alhaidari, "Exact solutions of dirac and schrödinger equations for a large class of power-law potentials at zero energy," International Journal of Modern Physics A, vol. 17, no. 30, p. 4551, 2002.

[2] J. W. Dabrowska, A. Khare, and U. Sukhatme, "Explicit wavefunctions for shape-invariant potentials by operator techniques," Journal of Physics A: Mathematical and General, vol. 21, p. L195, 1988.

[3] G. Levai, "A search for shape-invariant solvable potentials," Journal of Physics A: Mathematical and General, vol. 22, no. 6, pp. 689-702, 1989.

[4] R. De, R. Dut, and U. Sukhatme, "Mapping of shape invariant potentials under point canonical transformations," Journal of Physics A: Mathematical and General, vol. 25, pp. L843-L850, 1992.

[5] Y. Alhassid, F. Gursey, and F. Iachello, "Group theory approach to scattering. II. The euclidean connection," Annals of Physics, vol. 167 , no. 1 , p. 181, 1986.

[6] G. Levai, "Solvable potentials associated with su(1,1) algebras: a systematic study," Journal of Physics A: Mathematical and General, vol. 27, p. 3809, 1994.

[7] L. Infeld and T. E. Hull, "The factorization method," Reviews of Modern Physics, vol. 23, no. 1, pp. 21-68, 1951.

[8] E. Witten, "Dynamical breaking of supersymmetry," Nuclear Physics B, vol. 185, pp. 513-554, 1981.

[9] L. E. Gendenshtein, "Derivation of exact spectra of the schrodinger equation by means of supersymmetry," JETP Letters, vol. 38, pp. 356-359, 1983.

[10] F. Cooper, A. Khare, and U. Sukhatme, "Supersymmetry and quantum mechanics," Physics Reports, vol. 251, no. 5-6, pp. 267385, 1995.

[11] A. D. Alhaidari, "Relativistic extension of shape-invariant potentials," Journal of Physics A: Mathematical and General, vol. 34, p. 9827, 2001. 
[12] A. D. Alhaidari, "CORRIGENDUM: Relativistic extension of shape-invariant potentials," Journal of Physics A: Mathematical and General, vol. 35, p. 6207, 2002.

[13] A. D. Alhaidari, "Solution of the Relativistic Dirac-Morse Problem," Physical Review Letters, vol. 87, no. 24, 2001.

[14] H. Panahi and Z. Bakhshi, "Dirac equation and ground state of solvable potentials: supersymmetry method," International Journal of Theoretical Physics, vol. 50, no. 9, pp. 2811-2818, 2011.

[15] H. Panahi and Z. Bakhshi, "Dirac equation with positiondependent effective mass and solvable potentials in the Schrödinger equation," Journal of Physics A: Mathematical and Theoretical, vol. 44, p. 175304, 2011.

[16] L. H. Ryder, Quantum Field Theory, Cambrige University Press, Cambrige, UK, 1985.

[17] W. Greiner, Relativistic Quantum Mechanics, Springer-Verlag, Berlin, Germany, 1981.

[18] M. A. Jafarizadeh and H. Fakhri, "Calculation of the determinant of shape invariant operators," Physics Letters A, vol. 230, pp. 157-163, 1997.

[19] M. A. Jafarizadeh and H. Fakhri, "Supersymmetry and shape invariance in differential equations of mathematical physics," Physics letters A, vol. 230, pp. 164-170, 1997.

[20] M. A. Jafarizadeh and H. Fakhri, "Parasupersymmetry and shape invariance in differential equations of mathematical physics and quantum mechanics," Annals of Physics, vol. 262, no. 2, pp. $260-276,1998$. 

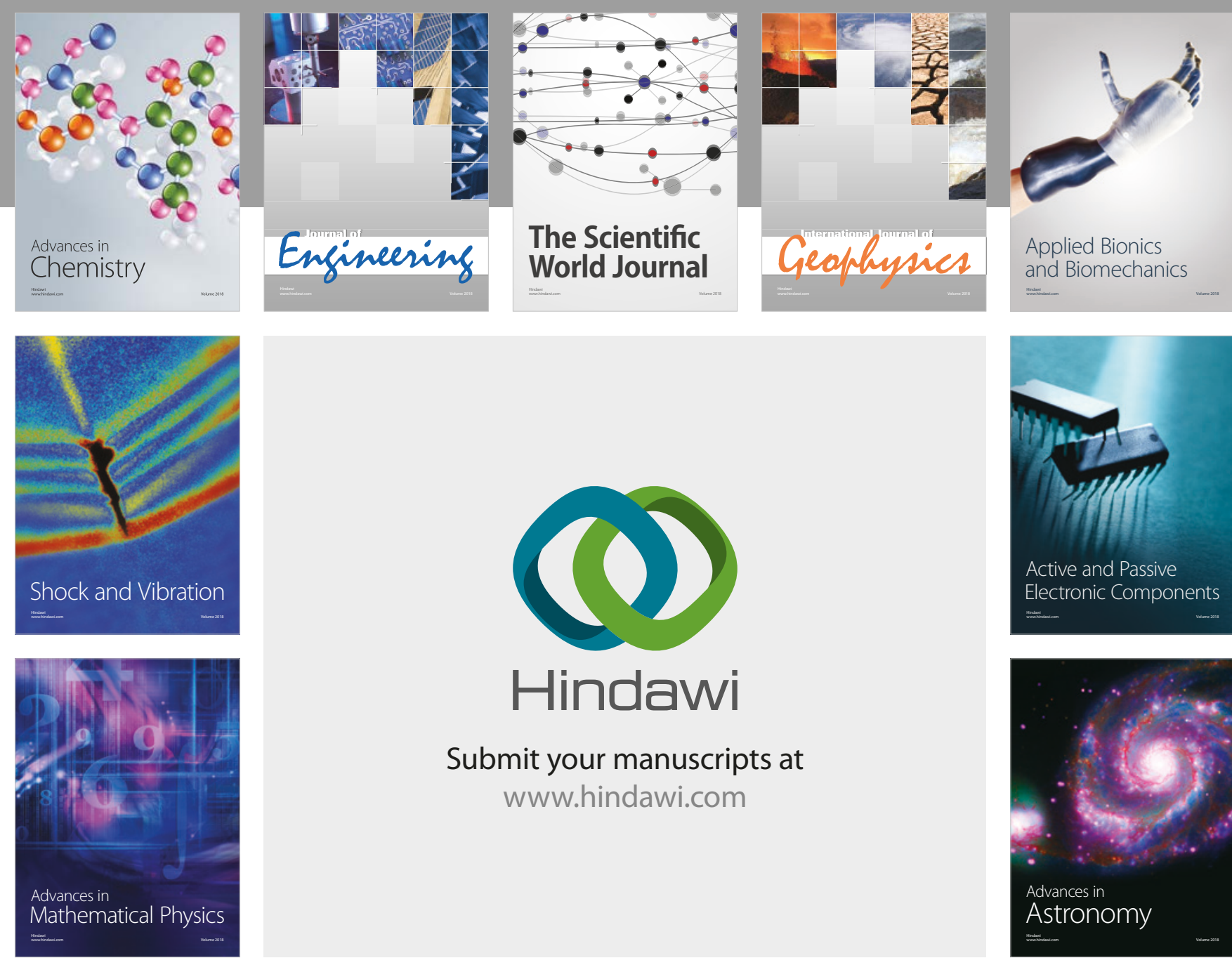

Submit your manuscripts at

www.hindawi.com

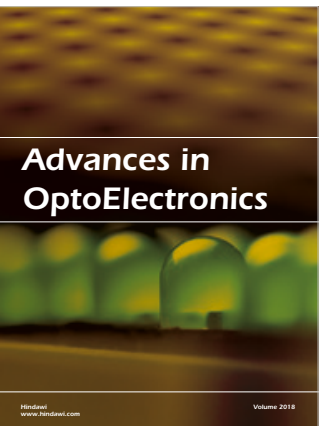

\section{Rotcting Machinery}
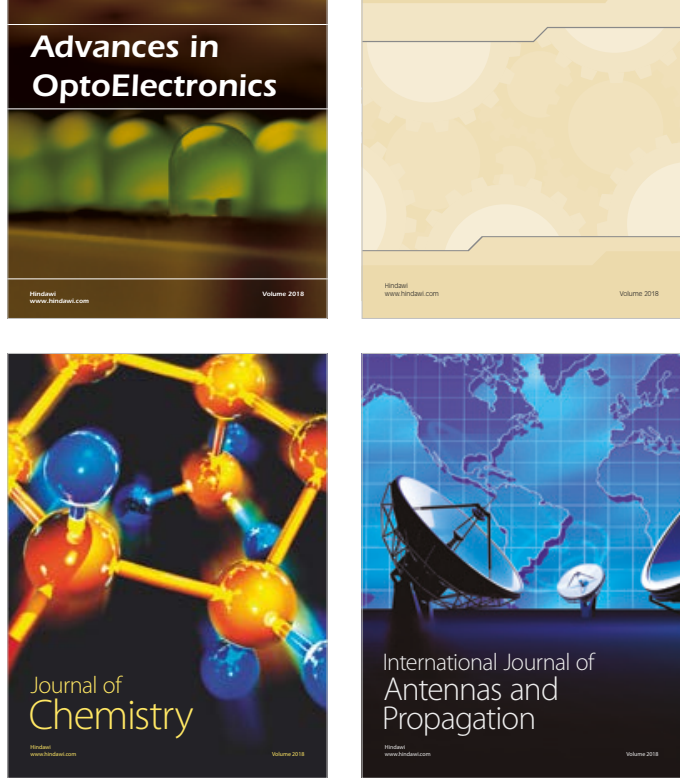

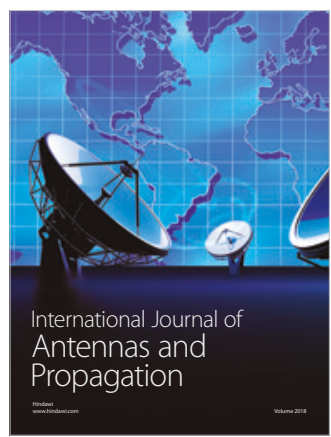

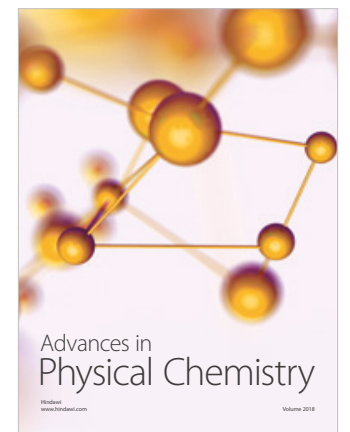

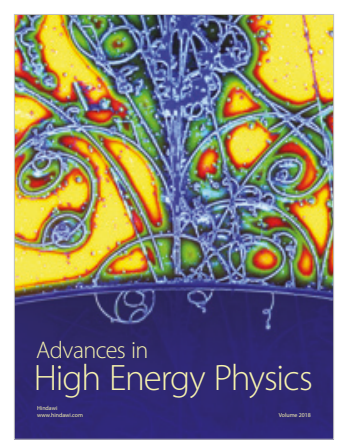

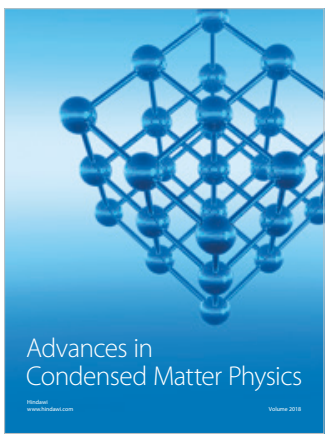

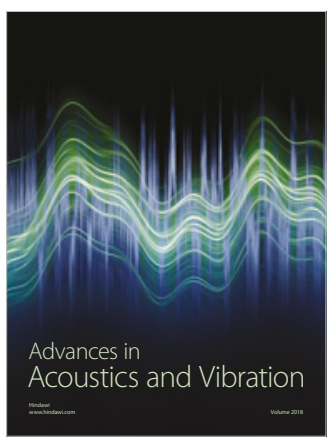

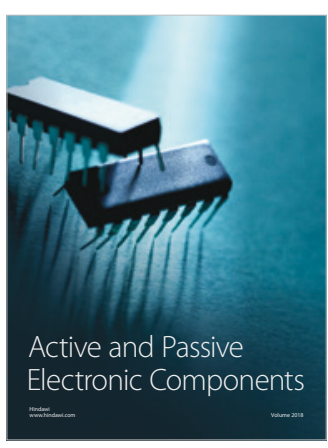
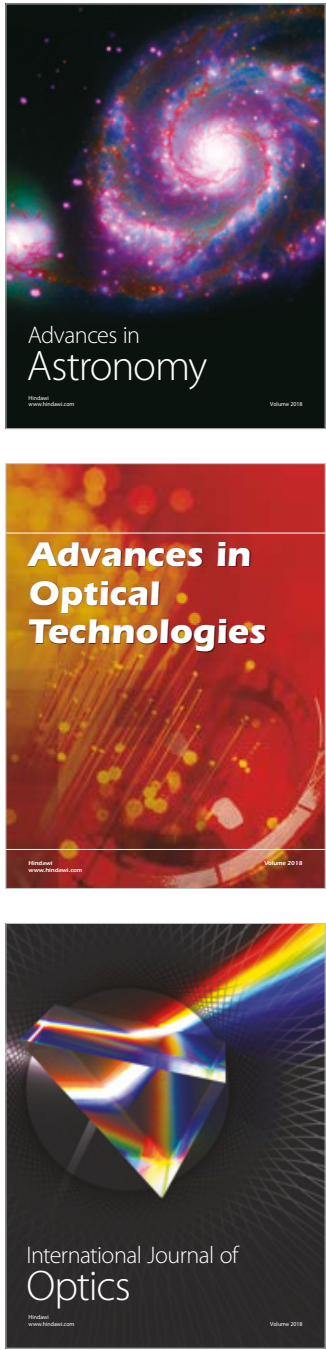